\title{
Epigenetics-related genes in prostate cancer: Expression profile in prostate cancer tissues, androgen-sensitive and -insensitive cell lines
}

\author{
ZAKI SHAIKHIBRAHIM, ANDREAS LINDSTROT, JACQUELINE OCHSENFAHRT, \\ KERSTIN FUCHS and NICOLAS WERNERT
}

Institute of Pathology, University Hospital Bonn, D-53127 Bonn, Germany

Received June 9, 2012; Accepted July 24, 2012

DOI: $10.3892 /$ ijmm.2012.1173

\begin{abstract}
Epigenetic changes have been suggested to drive prostate cancer ( $\mathrm{PCa}$ ) development and progression. Therefore, in this study, we aimed to identify novel epigenetics-related genes in PCa tissues, and to examine their expression in metastatic PCa cell lines. We analyzed the expression of epigeneticsrelated genes via a clustering analysis based on gene function in moderately and poorly differentiated PCa glands compared to normal glands of the peripheral zone (prostate proper) from PCa patients using Whole Human Genome Oligo Microarrays. Our analysis identified 12 epigenetics-related genes with a more than 2-fold increase or decrease in expression and a p-value $<0.01$. In moderately differentiated tumors compared to normal glands of the peripheral zone, we found the genes, TDRD1, IGF2, DICER1, ADARB1, HILS1, GLMN and TRIM27, to be upregulated, whereas TNRC6A and DGCR8 were found to be downregulated. In poorly differentiated tumors, we found TDRD1, $A D A R B$ and $R B M 3$ to be upregulated, whereas DGCR8, PIWIL2 and BC069781 were downregulated. Our analysis of the expression level for each gene in the metastatic androgen-sensitive $\mathrm{VCaP}$ and $\mathrm{LNCaP}$, and -insensitive PC3 and DU-145 PCa cell lines revealed differences in expression among the cell lines which may reflect the different biological properties of each cell line, and the potential role of each gene at different metastatic sites. The novel epigenetics-related genes that we identified in primary PCa tissues may provide further insight into the role that epigenetic changes play in PCa. Moreover, some of the genes that we identified may play important roles in primary $\mathrm{PCa}$ and metastasis, in primary $\mathrm{PCa}$ only, or in metastasis only. Follow-up studies are required to investigate the functional role and the role that the expression
\end{abstract}

Correspondence to: Professor Nicolas Wernert, Institute of Pathology, University Hospital Bonn, Sigmund-Freud-Str. 25, D-53127 Bonn, Germany

E-mail: nicolas.wernert@ukb.uni-bonn.de

Key words: epigenetics, prostate cancer, cell lines of these genes play in the outcome and progression of $\mathrm{PCa}$ using tissue microarrays.

\section{Introduction}

Prostate cancer ( $\mathrm{PCa})$ is one of the most common types of cancer affecting males in Western countries (1). Both steadily increasing age and the introduction of the serum marker prostate-specific antigen (PSA) have led to a steady increase in PCa incidence among Western populations $(2,3)$. The course of $\mathrm{PCa}$ is highly variable, from indolent carcinomas with a favorable prognosis requiring only watchful waiting, to highly lethal aggressive carcinomas with distant metastases needing systemic treatment $(4,5)$. Unfortunately, it is not possible to predict precisely based upon clinical data and biopsy alone the outcome of PCa in individual patients (3), as the disease is clinically heterogeneous and often multifocal with a clinical outcome that is difficult to predict (6,7). As a result, tremendous efforts and resources have been invested over the years with the ultimate aim of identifying molecular markers possessing independent prognostic clues or serving as potential therapeutic targets in $\mathrm{PCa}$.

Similar to other cancers, the development and progression of PCa is driven by the interplay of genetic as well as epigenetic changes (5). Epigenetic changes result in the modification of gene expression and function without changes in the DNA sequence (8). They include both the direct methylation of the $\mathrm{CpG}$ islands (9-11) and histone modifications (e.g., acetylation, ADP-ribosylation, ubiquination, phosphorylation and methylation) $(9,11)$. The extent of histone methylation may vary (mono- to trimethylation) and the location of the modification can determine whether a gene will be silenced or transcribed (9). Both DNA methylation and histone modifications have been implicated in $\mathrm{PCa}$, and have been proposed to influence tumor formation (11). For example, more than 50 hypermethylated genes have been found in $\mathrm{PCa}$, and approximately a dozen of these genes have been found to be consistently affected in the majority of cases examined (5). Many of the genes that have been found to be methylated in $\mathrm{PCa}$ are known to affect cell cycle control, tumor invasion and signal transduction (11). 
Due to the crucial role that epigenetic changes play in $\mathrm{PCa}$ and their potential use as diagnostic and prognostic markers in the future, we in this study, aimed to identify novel epigeneticsrelated genes in primary $\mathrm{PCa}$ tissues, and to examine their expression in metastatic androgen-sensitive and -insensitive PCa cell lines.

\section{Materials and methods}

Ethics statement. This study was approved by the Ethics Eeview Board of the University of Bonn/University Hospital, Bonn, Germany, according to the principles expressed in the Declaration of Helsinki. Written informed consents were obtained from all participants.

Microarray raw data. The microarray raw data were deposited in the GEO database under accession number: GSE28615 and we confirm that all details are MIAME compliant.

Processing of human prostatectomy specimens. Prostate tissue samples were obtained from PCa patients immediately after radical prostatectomy. Fresh tissue samples $(0.5 \times 0.5 \times 0.3 \mathrm{~cm})$ were obtained from the peripheral zones (prostate proper) of 5 moderately differentiated (Gleason scores 6 and 7a) PCa patients, 4 poorly differentiated (Gleason scores 8 and 9) $\mathrm{PCa}$ patients, as well as from 10 normal peripheral zones of $\mathrm{PCa}$ patients. The tissues were shock-frozen in liquid nitrogen with ice-cold isopentane. Frozen sections (6- $\mu \mathrm{m}$ thick) were cut from the samples using a cryotome (Leica, Germany) and mounted on membrane-coated slides (Membrane Slides, $1 \mathrm{~mm}$ PEN, Zeiss, Jena, Germany) for subsequent laser microdissection. One section was mounted on conventional slides and stained with hematoxylin and eosin (H\&E) for diagnostic evaluation by an experienced pathologist. Laser microdissection was performed as previously described (12-14). The frozen sections were then dried for $2 \mathrm{~min}$ in the cryotome, washed for 2 min with $70 \%$ ethanol in DEPC-treated water and stained for $30 \mathrm{sec}$ in $1 \%$ cresyl violet diluted in 50\% ethanol-DEPCtreated water. The slides were then washed briefly in 70 and $100 \%$ ethanol, dried for $10 \mathrm{~min}$ and stored at $-80^{\circ} \mathrm{C}$ until use for laser microdissection of normal glands and the stroma between them.

Quality control. RNA quality was measured from each patient before laser microdissection using laser capture microscopy (LCM). The section was washed from the slide with $600 \mu \mathrm{l}$ buffer RLT $+2 \mu \mathrm{M}$ DTT (RNeasy Mini kit, Qiagen, Hilden, Germany) and vortexed for $30 \mathrm{sec}$. RNA extraction was performed as described by the manufacturer. The recommended DNase digestion was made with RNasefree DNase set (Qiagen). RNA quality was measured with the Agilent Bioanalyzer 2100 (Agilent Technologies, Santa Clara, CA, USA). Samples with a RIN factor $>6$ were used for LCM.

$L C M$. The cresyl violet-stained sections were visualized with an Axio Observer.Z1 microscope (Zeiss) with an installed Palm MicroBeam (Zeiss). LCM was performed under x10 objective lenses. The glands from carcinomas and the normal peripheral zones were isolated from the stroma by laser microdissection and collected in 200- $\mu 1$ adhesive cap tubes (Zeiss).

Microarray analysis of RNA isolated from laser-microdisected moderately and poorly differentiated prostate carcinoma glands, as well as from normal glands of PCa patients. RNA was isolated from laser-microdisected moderately and poorly differentiated PCa glands, and from normal glands from $\mathrm{PCa}$ patients using the RNeasy Micro kit (Qiagen) as described by the manufacturer. The recommend DNase digestion was included with the RNase-free DNase set (Qiagen). The amount of isolated RNA was measured with the Nanodrop photometer (Thermo Fisher Scientific, Hudson, NH, USA). Thereafter, an equal amount of RNA from the normal peripheral glands, moderately differentiated glands and poorly differentiated glands was pooled to final concentrations of $300 \mathrm{ng}$ of RNA, respectively. These pools were then sent to Miltenyi Biotec (Bergisch Gladbach, Germany) for the microarray analysis and bioinformatics interpretation. The RNA was labelled with Cy3 and hybridized on the Whole Human Genome Oligo Microarray 4x44K (Agilent Technologies) according to the manufacturer's instructions. The microarray results were then validated by qRT-PCR of a subset of genes.

RNA isolation from PCa cell lines and cDNA synthesis. RNA was isolated from the PCa cell lines, VCaP, DU-145, LNCaP and PC3 using the RNeasy mini kit (Qiagen) as described by the manufacturer. The amount of isolated RNA was then measured with the ND1000 Nanodrop (Peqlab, Wilmington, DE, USA). Reverse transcription of total RNA was performed with the SuperScript III First-Strand Synthesis SuperMix as described by the manufacturer (Invitrogen, Carlsbad, CA, USA).

Polymerase chain reaction (PCR). PCR was performed using the DNA Engine Peltier thermal cycler (Bio-Rad, Munich, Germany) to analyze the expression of $R B M 3$, GLMN, BC068781, TNRC6A, TDRD1, DGCR8, HILS1, TRIM27, DICER1, PIWIL2, IGF2 and ADARB1 and the housekeeping gene, GAPDH. The primers and sequences for all the latter genes are listed in Table I. All PCR products were analyzed on $2 \%$ agarose gels that were stained with ethidium bromide.

\section{Results}

Gene expression analysis of moderately and poorly differentiated prostate carcinoma glands compared to normal glands of the the peripheral zone (prostate proper) from $\mathrm{PCa}$ patients identifies epigenetics-related genes using Whole Human Genome Oligo Microarrays. To identify epigeneticsrelated genes in $\mathrm{PCa}$, we analyzed the expression profile of epigenetics-related genes in the moderately and poorly differentiated PCa glands compared to normal glands of the peripheral zone (prostate proper) from PCa patients using 2 Whole Human Genome Oligo Microarrays. Genes with a more than 2-fold increase or decrease in expression and a p-value $<0.01$ were considered significant. A clustering analysis based on gene function identified 12 epigenetics-related genes (Tables II-V). 
Table I. The sequences of primers used in the RT-PCR analysis.

\begin{tabular}{lll}
\hline Short Name & \multicolumn{1}{c}{ Left primer } & \multicolumn{1}{c}{ Right primer } \\
\hline$R B M 3$ & GCTGCACCGAAGCATCTTAT & CCAAACTTGCCAGAACCAAT \\
GLMN & CAGCAATTGGACACCCTTTC & TGCCCCATATTAAACTGCAA \\
BC069781 & TTTGTCAGGCACAGATGCTT & CGATGCTGATTGAGGTTGTG \\
$T D R D 1$ & TTGAATCATGCAGGCCAATA & AGTGCAAAGGGAAAAGCTCA \\
$D G C R 8$ & GCTCCACAGCATGTCAAAGA & AGCCCAAATGGCTATTTCCT \\
$H I L S 1$ & GGGGGTGAGAGTGCTGATAA & GGGAAATTCAAGGCCTCTTC \\
$T R I M 27$ & GCTCAAGGTCAAGAGGCAAC & TGCCTCATTAATTGCAGTGG \\
$D I C E R 1$ & TCAGTGGACGTGACTCTGGA & CCACCTTTTCTGCACACTGA \\
$P I W I L 2$ & AAGGAAGCTGGCAAACAAGA & AAAACGAACCACCAAGTTGC \\
$I G F 2$ & TCTATGGGGCCATCAAGAAG & CCATCCCGATCACCATTAAC \\
ADARB 1 & TCCTCCCTGGACAATCAGAC & AGAAGCACCAGCATCGACTT \\
GAPDH & ACCTCCACCAAGCTCAGAGA & ATGCTGTGGGGTAAGGTCTG \\
\hline
\end{tabular}

Table II. Genes upregulated in moderately differentiated tumors compared to normal glands of the peripheral zone.

\begin{tabular}{llr}
\hline Short name & & \multicolumn{1}{c}{ Long name } \\
TDRD1 & Tudor domain-containing 1 & Fold change \\
IGF2 & Insulin-like growth factor 2 (somatomedin A); INS-IGF2 readthrough transcript & 17.2 \\
DICER1 & Dicer 1, ribonuclease type III & 6.6 \\
ADARB1 & Adenosine deaminase, RNA-specific & 4.8 \\
HILS1 & Histone linker H1 domain, spermatid-specific 1 & 4.6 \\
GLMN & Glomulin, FKBP associated protein & 3.8 \\
TRIM27 & Tripartite motif-containing 27 & 2.9 \\
\hline
\end{tabular}

Table III. Genes downregulated in moderately differentiated tumors compared to normal glands of the peripheral zone.

\begin{tabular}{llc}
\hline Short name & \multicolumn{1}{c}{ Long name } & Fold change \\
\hline TNRC6A & $\begin{array}{l}\text { Trinucleotide repeat } \\
\text { containing 6A }\end{array}$ & -2.9 \\
DGCR8 & $\begin{array}{l}\text { DiGeorge syndrome } \\
\text { critical region gene } 8\end{array}$ & -3.4 \\
\hline
\end{tabular}

The epigenetics-related genes, TDRD1, IGF2, DICER1, $A D A R B 1, H I L S 1, G L M N$ and TRIM27, were found to be upregulated in the moderately differentiated tumors compared to the normal glands of the peripheral zone (Table II), whereas TNRC6A and DGCR8 were found to be downregulated (Table III).

In the poorly differentiated tumors compared to the normal glands of the peripheral zone, the epigenetics-related genes, $T D R D 1 A D A R B$ and $R B M 3$, were found to be upregulated
Table IV. Genes upregulated in poorly differentiated tumors compared to normal glands of the peripheral zone.

\begin{tabular}{llc}
\hline Short name & \multicolumn{1}{c}{ Long name } & Fold change \\
\hline TDRD1 & Tudor domain containing 1 & 8.5 \\
ADARB1 & Adenosine deaminase, & 5.3 \\
& RNA-specific & \\
RBM3 & RNA binding motif (RNP1, RRM) & 2.4 \\
& protein 3; hypothetical LOC729275 & \\
\hline
\end{tabular}

(Table IV), whereas DGCR8, PIWIL2 and BC069781 were found to be downregulated (Table V).

Gene expression of epigenetics-related genes identified from PCa tissues in androgen-sensitive and-insensitive PCa cell lines. The epigenetics-related genes identified in PCa tissues (Tables II-V) were examined for their expression in the androgen-sensitive $\mathrm{VCaP}$ and $\mathrm{LNCaP}$ and -insensitive $\mathrm{PC} 3$ and DU-145 PCa cell lines (Fig. 1). The expression level for each 
Table V. Genes downregulated in poorly differentiated tumors compared to normal glands of the peripheral zone.

\begin{tabular}{llc}
\hline Short name & \multicolumn{1}{c}{ Long name } & Fold change \\
\hline DGCR8 & $\begin{array}{l}\text { DiGeorge syndrome } \\
\text { critical region gene } 8\end{array}$ & -3.8 \\
PIWIL2 & Piwi-like 2 (Drosophila) & -3.1 \\
BC069781 & SMAD family member 1 & -3.0 \\
\hline
\end{tabular}

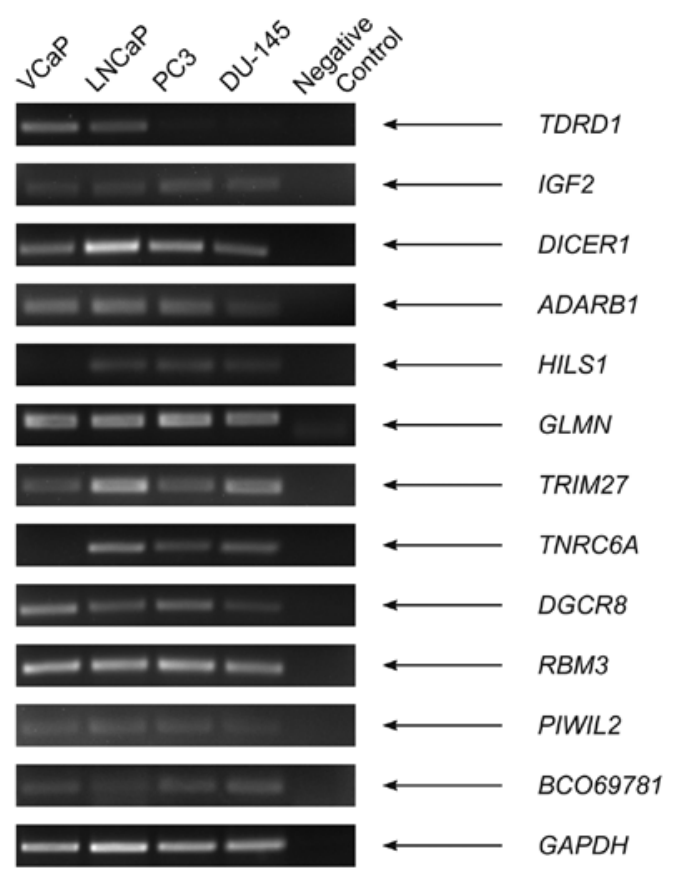

Figure 1. mRNA expression of epigenetics-related genes in prostate cancer cell lines. RT-PCR analysis of epigenetics-related genes in the androgendependent $\mathrm{VCaP}$ and $\mathrm{LNCaP}$, and the androgen-independent $\mathrm{PC} 3$ and DU-145 prostate cancer cell lines. The gene GAPDH was used as the loading control, and amplification without a template was used as the negative control.

gene differed among the 4 cell lines examined. For instance, TDRD1 was expressed only in the androgen-sensitive PCa cell lines, but not in the insensitive ones. HILS1 and TNRC6A were expressed in all the cell lines expect in the androgen-sensitive VCaP cell line. We found PIWIL2 and BCO69781 to be very weakly expressed in all the cell lines examined, whereas $I G F 2$, DICER1, ADARB1, GLMN, TRIM27, DGCR8 and RBM3 were expressed in all 4 cell lines at different levels.

\section{Discussion}

Genetic and epigenetic changes have been suggested to drive PCa development and progression (5). For instance, DNA methylation and histone modifications have been suggested to influence PCa tumor formation (11). Hypermethylated genes have been found in PCa (5), and many of them are known to affect various cellular processes, such as cell cycle control, tumor invasion and signal transduction (11). Therefore, due to the crucial role that epigenetic changes play in $\mathrm{PCa}$ and their potential use as diagnostic and prognostic markers in the future, in this study, we aimed to identify novel epigeneticsrelated genes in PCa tissues, and to examine their expression in metastatic androgen-sensitive and -insensitive PCa cell lines.

To identify epigenetics-related genes in PCa, we analyzed the expression profile of epigenetics-related genes via a clustering analysis based on gene function in the moderately and poorly differentiated PCa glands compared to normal glands of the peripheral zone (prostate proper) from $\mathrm{PCa}$ patients using Whole Human Genome Oligo Microarrays. Our analysis identified 12 epigenetics-related genes with a more than 2-fold increase or decrease in expression and a p-value $<0.01$ (Tables II-V).

In the moderately differentiated tumors compared to normal glands of the peripheral zone, we found the epigenetics-related genes, TDRD1, IGF2, DICER1, ADARB1, HILS1, GLMN and TRIM27, to be upregulated (Table II), whereas TNRC6A and $D G C R 8$ were found to be downregulated (Table III). In poorly differentiated tumors, we found TDRD1, ADARB and RBM3 to be upregulated (Table IV), whereas DGCR8, PIWIL2 and BC069781 were downregulated (Table V).

Our literature search of these epigenetics-related genes revealed 5/12 of these genes to be implicated in PCa, while the rest are either involved in other types of tumors or have not yet been shown to be involved in cancer. Briefly, DICERI which we found to be upregulated in the tumors compared to normal glands, has previously been reported to be upregulated in PCa (15). Of note, the overexpression of DICERI has been suggested to predict poor survival in colorectal cancer (16). As regards $I G F 2$, dysregulation has been suggested to be an early change in $\mathrm{PCa}$, and inactivation has been associated with cancer progression (17). TNRC6A has been reported to be expressed in $55 \%$ of prostatic intraepithelial neoplasia (PIN) and $63.6 \%$ of PCa cases (18). Concerning RBM3, a high protein expression in $\mathrm{PCa}$ has been shown to independently predict a reduced risk of biochemical recurrence and disease progression (19). PIWIL2 has been reported to be expressed in many tumors including $\mathrm{PCa}$, and has been shown to inhibit apoptosis through the activation of the Stat3/Bcl-xL pathway (20). TDRD1, which is known to be specifically expressed in the testis is expressed in both hepatocellular carcinoma and non-tumorous liver tissues (21). ADARB1, an editing mediating enzyme, has been detected at reduced RNA levels in brain tumors, and the overexpression of $A D A R B 1$ in a glioblastoma multiforme cell line has been shown to lead to decreased proliferation (22). DGCR8 is a double-cysteine-ligated heme protein (23) and is part of a multi-subunit protein complex known as the microprocessor complex, which has been shown to be necessary and sufficient for processing miRNA precursor RNAs (24). Finally, to our knowledge, there are no cancer studies to date reporting on HILS1, GLMN, TRIM27 and $B C 069781$.

In order to gain insight into the potential role in metastasis of the 12 epigenetics-related genes that we identified in primary $\mathrm{PCa}$, we examined the expression profile of the latter genes in the 4 well characterized androgen-sensitive $\mathrm{VCaP}$ and LNCaP, and -insensitive PC3 and DU-145 PCa cell lines (Fig. 1). The 4 cell lines were derived from different origins. DU-145, PC3, LNCaP and VCaP cells were derived from brain metastasis, advanced androgen independent bone metastasis, 
supraclavicular lymph node metastasis and a metastatic lesion to a lumbar vertebral body of a patient with hormone refractory $\mathrm{PCa}$, respectively. Our analysis revealed the expression level for each gene to be different among the 4 cell lines examined (Fig. 1). For example, TDRD1 was only expressed in the androgen-sensitive PCa cell lines, and not in the insensitive cell lines. HILS1 and TNRC6A were expressed in all the cell lines except in the androgen-sensitive $\mathrm{VCaP}$ cell line. PIWIL2 and BCO69781 were weakly expressed in all the cell lines examined, whereas IGF2, DICER1, ADARB1, GLMN, TRIM27, DGCR8 and RBM3 were expressed in all 4 cell lines at different levels. The differences in expression pattern of these 12 genes among the 4 cell lines may reflect the different biological properties of each cell line, and the potential role each gene may play at different metastatic sites.

Taken together, in this study, we identify novel epigeneticsrelated genes in primary $\mathrm{PCa}$ tissues, which we believe are highly significant due to the crucial role that epigenetic changes play in PCa. It is likely, based on our gene expression profile data, that some of the genes that we identified may play important roles in primary $\mathrm{PCa}$ and metastasis, in primary only, or in metastasis only. Follow-up studies are required to investigate the functional role and the role that the expression of these genes play in the outcome and progression of $\mathrm{PCa}$ using tissue microarrays comprised of primary PCa samples, lymph node and distant metastases.

\section{Acknowledgements}

The present study was supported by the DFG (Deutsche Forschungsgemeinschaft; German Research Association, grant no. WE 1104/11-1, and the Deutsche Krebshilfe (German Cancer Aid, grant no. 107827).

\section{References}

1. Jemal A, Siegel R, Xu J and Ward E: Cancer statistics, 2010. CA Cancer J Clin 60: 277-300, 2010.

2. Draisma G, Etzioni R, Tsodikov A, et al: Lead time and overdiagnosis in prostate-specific antigen screening: importance of methods and context. J Natl Cancer Inst 101: 374-383, 2009.

3. Park JY: Promoter hypermethylation in prostate cancer. Cancer Control 17: 245-255, 2010.

4. Wu JN and Dall'Era MA: Active surveillance for localized prostate cancer - current practices and recommendations. Sci World J 10: 2352-2361, 2010.

5. Schulz WA and Hoffmann MJ: Epigenetic mechanisms in the biology of prostate cancer. Semin Cancer Biol 19: 172-180, 2009.
6. Arora R, Koch MO, Eble JN, Ulbright TM, Li L and Cheng L: Heterogeneity of Gleason grade in multifocal adenocarcinoma of the prostate. Cancer 100: 2362-2366, 2004.

7. Ruijter ET, Van de Kaa CA, Schalken JA, Debruyne FM and Ruiter DJ: Histological grade heterogeneity in multifocal prostate cancer. Biological and clinical implications. J Pathol 180: 295-299, 1996.

8. Rennie PS and Nelson CC: Epigenetic mechanisms for progression of prostate cancer. Cancer Metastasis Rev 17: 401-409, 1998.

9. Iacobuzio-Donahue CA: Epigenetic changes in cancer. Annu Rev Pathol 4: 229-249, 2009.

10. Perry AS, Watson RW, Lawler M and Hollywood D: The epigenome as a therapeutic target in prostate cancer. Nat Rev Urol 7: 668-680, 2010.

11. Joshua AM, Evans A, Van der Kwast T, et al: Prostatic preneoplasia and beyond. Biochim Biophys Acta 1785: 156-181, 2008.

12. Wellmann A, Wollscheid V, Lu H, et al: Analysis of microdissected prostate tissue with ProteinChip arrays - a way to new insights into carcinogenesis and to diagnostic tools. Int J Mol Med 9: 341-347, 2002

13. Okuducu AF, Janzen V, Hahne JC, Ko Y and Wernert N: Influence of histochemical stains on quantitative gene expression analysis after laser-assisted microdissection. Int J Mol Med 11: 449-453, 2003

14. Okuducu AF, Hahne JC, Von Deimling A and Wernert N: Laser-assisted microdissection, techniques and applications in pathology (Review). Int J Mol Med 15: 763-769, 2005.

15. Chiosea S, Jelezcova E, Chandran U, et al: Up-regulation of dicer, a component of the MicroRNA machinery, in prostate adenocarcinoma. Am J Pathol 169: 1812-1820, 2006.

16. Faber C, Horst D, Hlubek F and Kirchner T: Overexpression of Dicer predicts poor survival in colorectal cancer. Eur J Cancer 47: 1414-1419, 2011.

17. Ribarska T, Bastian KM, Koch A and Schulz WA: Specific changes in the expression of imprinted genes in prostate cancerimplications for cancer progression and epigenetic regulation. Asian J Androl 14: 436-450, 2012.

18. Yoo NJ, Hur SY, Kim MS, Lee JY and Lee SH: Immunohistochemical analysis of RNA-induced silencing complex-related proteins AGO2 and TNRC6A in prostate and esophageal cancers. APMIS 118: 271-276, 2010.

19. Jonsson L, Gaber A, Ulmert D, Uhlen M, Bjartell A and Jirstrom K: High RBM3 expression in prostate cancer independently predicts a reduced risk of biochemical recurrence and disease progression. Diagn Pathol 6: 91, 2011.

20. Lee JH, Schutte D, Wulf G, et al: Stem-cell protein Piwil2 is widely expressed in tumors and inhibits apoptosis through activation of Stat3/Bcl-XL pathway. Hum Mol Genet 15: 201-211, 2006.

21. Yoon $\mathrm{H}$, Lee $\mathrm{H}, \mathrm{Kim} \mathrm{HJ}$, et al: Tudor domain-containing protein 4 as a potential cancer/testis antigen in liver cancer. Tohoku J Exp Med 224: 41-46, 2011.

22. Paz N, Levanon EY, Amariglio N, et al: Altered adenosineto-inosine RNA editing in human cancer. Genome Res 17: 1586-1595, 2007

23. Barr I, Smith AT, Senturia R, et al: DiGeorge critical region 8 (DGCR8) is a double-cysteine-ligated heme protein. J Biol Chem 286: 16716-16725, 2011.

24. Gregory RI and Shiekhattar R: MicroRNA biogenesis and cancer. Cancer Res 65: 3509-3512, 2005. 